\title{
Rupture of the muscle-tendon complex in tensile test. Comparison between experimentations and discrete element modeling
}

\author{
A. Roux ${ }^{a, b}$, T.-X. Haen ${ }^{a}$, J. Lecompte ${ }^{a}$, I. lordanoff ${ }^{b}$ and S. Laporte ${ }^{a}$

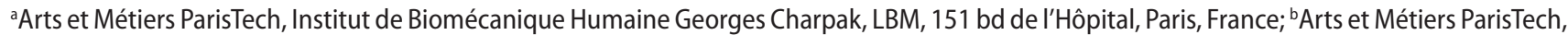 \\ I2M, Esplanade des Arts et Métiers, Talence, France
}

KEYWORDS Muscle-tendon complex; discrete element method; rupture

\section{Introduction}

Tear of the muscle-tendon complex (MTC) is common sports-relatedinjury. Musclesaremorepronetotearduring eccentric exercises when muscle contraction is combined with excessive stretching (Uchiyama et al. 2011). Following a tear of a muscle, functional impairment appeared as the result of altered MTC's mechanical properties. However, the mechanisms leading to such injury are still unclear as well as structures involved that could provide a rational basis for injury prevention and/or rehabilitation (Uchiyama et al. 2011). To better understand the MTC's tear, a model of the MTC's rupture behavior in tensile tests is created. The aim of this study was to compare this model with experimental findings, in order to validate our model.

\section{Methods}

\subsection{Computational modeling}

The MTC's model was developed in discrete element method (DEM) (Roux et al. 2014) (Figure 1(a)) with GranOO software (www.granoo.org, I2M, France). All MTC's components (muscle, tendon, epimysium, myo-tendinous junction (MTJ), and extra cellular matrix) are modeled with simple discrete elements linked with springs. Stiffness of each element was related to discrete elements' cross-sectional area, initial length of links, and corresponding elastic modulus. Fibers were built with spherical discrete elements linked with springs. The extra cellular matrix was computed using springs between fibers. Tendon's fibers were built with respect to muscle fibers architecture. The MTJ was represented by multi-links between tendon and muscle. Mechanical properties of MTC's components are addressed thanks to literature values. MTC is fixed on its lower extremity, and the upper extremity is subjected to a linear displacement until rupture. Rupture's criterion is an elongation criterion for all MTC's springs. Force/ displacement curves and discrete elements' stress are computed during the tensile test. Rupture localization is also studied.

\subsection{Experimental testing}

Twelve lower limbs of fresh frozen human cadavers (age $=84 \pm 5$ years, mean \pm SD) are examined. Achilles Tendons and Triceps surae muscles are isolated, with their bone insertion for fixation in testing machine. A break strength testing at $2 \mathrm{~mm} / \mathrm{s}$ is performed on the anatomical preparations. Same boundary conditions as DEM tensile tests are applied to experimental tests (Figure 1(b)).

\section{Results and discussion}

The computed and experimental force/displacement curves display a classical hyper-elastic behavior until rupture. The numerical behavior is obtained with linear springs, and thanks to the MTC's structural effect. In MTC's DEM model, rupture is located in the MTJ with a delamination of muscle's fibers in this area. Stress concentration is located in the MTJ's area, and a high displacement of links between elements is imposed at the MTJ's level. Rupture is caused by delamination: fibers are broken one by one until complete deterioration of the MTJ (Figure 2(a) and (b)).

In experimental tests, rupture of $\{$ Achilles Tendon + Triceps surae muscle occurs in 11 cases at the MTJ's level, and a tendon avulsion is observed in one case. Experimental and computed force/displacement relationships are in agreement. A first high delamination is observed; then a second rupture 
(a)

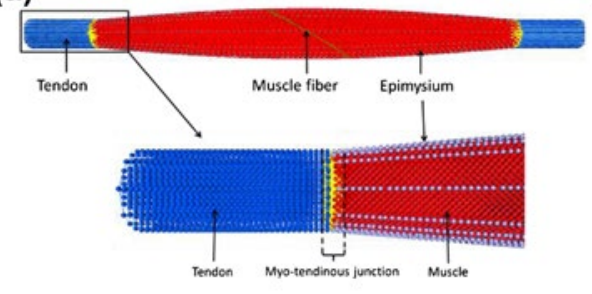

(b)

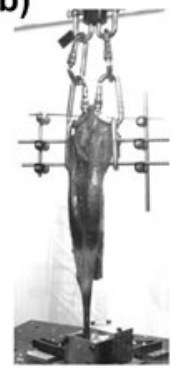

Figure 1. (a) MTC's geometry in DEM and (b) experimental setup on \{Achilles Tendon + Triceps surae muscle\}.
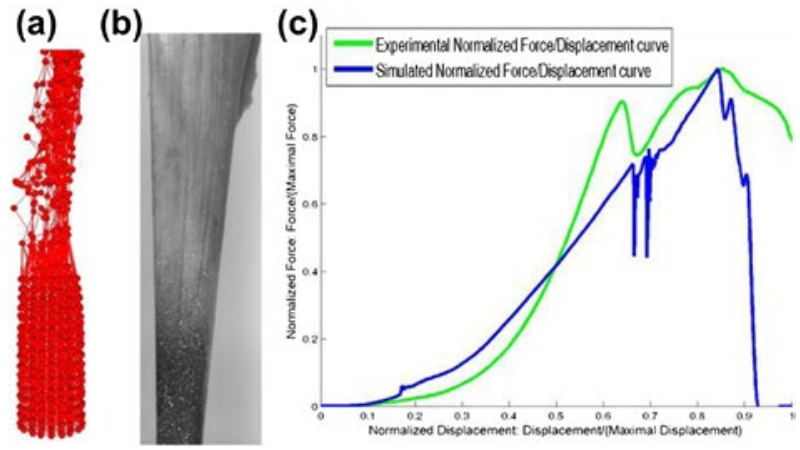

Figure 2. (a) Rupture in the myo-tendinous junction of the MTC in tensile test with DEM, (b) delamination of the MTJ during the experimental destructive tensile test, and (c) comparison between experimental and simulated force/displacement curves.

highlights the global delamination of the MTJ with a wrenching of muscle's fibers (Figure 2(c)). Even if they are muscles from humans' cadavers, data can be used for the validation of numerical models.

Those results are also in agreement with literature concerning the percentage of rupture in the MTJ's site (80\%) (Ilaslan et al. 2007). Rupture model is in

agreement with experimental data on the aspect of force/ displacement curve, on the localization of the rupture site (Law and Lightner 1993; Bianchi et al. 2006) and with the fibers' delamination of the MTC. Rupture is located in the MTJ's area of the MTC, as described in the literature, because MTJ was between tendon (stiff) and muscle (soft). The stiffness's variation leads this area to a stress concentration during the tensile test, therefore a rupture. DEM enables to model fibers' delamination and rupture for MTC, with high displacement and with simple microscopic mechanical law.

\section{Conclusions}

The DEM seems to be a promising method to model the rupture response of MTC, in agreement with experimental data. The next step will be to improve the model with contraction, in order to model the tear of the MTC.

\section{References}

Bianchi S, Poletti P-A, Martinoli C, Abdelwahab IF. 2006. Ultrasound appearance of tendon tears. Part 2: lower extremity and myotendinous tears. Skeletal Radiol. 35: 63-77.

Ilaslan H, Iannotti JP, Recht MP. 2007. Deltoid muscle and tendon tears in patients with chronic rotator cuff tears. Skeletal Radiol. 36:503-507.

Law DJ, Lightner VA. 1993. Divalent cation-dependent adhesion at the myotendinous junction: ultrastructure and mechanics of failure. J Muscle Res Cell Motil. $14: 173-185$.

Roux A, Lecompte J, Gras L-L, Laporte S, Iordanoff I. 2014. Tensile response of the muscle-tendon complex using discrete element model. Comput Methods Biomech Biomed Eng. 17:134-135.

Uchiyama Y, Miyazaki S, Tamaki T, Shimpuku E, Handa A, Omi H, Mochida J. 2011. Clinical results of a surgical technique using endobuttons for complete tendon tear of pectoralis major muscle: report of five cases. Sports Med Arthrosc Rehabil Ther Technol. 3:20-1-20-9. 